\title{
Momentum Compaction and Phase Slip Factor
}

\author{
K.Y. Ng \\ Fermilab, Batavia, IL 60510
}

(October, 2010)

\begin{abstract}
Section 2.3.11 of the Handbook of Accelerator Physics and Engineering on Landau damping is updated. The slip factor and its higher orders are given in terms of the various orders of the momentum compaction. With the aid of a simplified FODO lattice, formulas are given for the alteration of the lower orders of the momentum compaction by various higher multipole magnets. The transition to isochronicity is next demonstrated. Formulas are given for the extraction of the first three orders of the slip factor from the measurement of the synchrotron tune while changing the rf frequency. Finally bunch-length compression experiments in semi-isochronous rings are reported .
\end{abstract}

Submitted to 3rd edition of Handbook of Accelerator Physics and Engineering 


\subsubsection{Momentum Compaction and Phase Slip Factor \\ K.Y. Ng, FNAL}

The phase slip factor $\eta$ is the relative slip in revolution period $T$ for a particle with fractional offmomentum $\delta=\Delta p / p_{0}$, i.e. $\Delta T / T_{0}=\eta \delta$, where the subscript zero stands for on-momentum. The various orders of momentum-compaction factor $\alpha_{i}$ give the relative increase in closed-orbit length $C$ for an off-momentum particle, or $\Delta C / C_{0}=$ $\sum_{i=0}^{\infty} \alpha_{i} \delta^{i+1}$. With $\eta=\sum_{i=0}^{\infty} \eta_{i} \delta^{i}$, we have [1]

$$
\begin{aligned}
& \eta_{i}=\alpha_{i}-\frac{\eta_{i-1}}{\gamma_{0}^{2}}+\frac{3 \beta_{0}^{2} \eta_{i-2}}{2 \gamma_{0}^{2}}+\frac{\left(1-5 \beta_{0}^{2}\right) \beta_{0}^{2} \eta_{i-3}}{2 \gamma_{0}^{2}}- \\
& -\frac{5\left(3-7 \beta_{0}^{2}\right) \beta_{0}^{4} \eta_{i-4}}{8 \gamma_{0}^{2}}+\cdots, \eta_{i}= \begin{cases}1, & i=-1, \\
0, & i<-1,\end{cases}
\end{aligned}
$$

where $\beta_{0}$ and $\gamma_{0}$ are the on-momentum Lorentz factors. The transition gamma is defined as $\gamma_{t}=$ $\sqrt{1 / \alpha_{0}}$. To lowest order, all off-momentum particles have the same transition gamma when $\alpha_{1} / \alpha_{0} \approx-\frac{1}{2}$, and cross transition at the same time when $\alpha_{1} / \alpha_{0} \approx-\frac{3}{2}$.

For a FODO lattice with thin quadruples of length $\ell$ and strength $B^{\prime} \ell /(B \rho)= \pm S / L$, where $L$ is the half cell length with dipole bending angle $\theta$, we have [2, 3] (see also Eq.(8), Sec.2.2.3)

$$
\begin{gathered}
\alpha_{0} \approx 1-\frac{S\left(\hat{D}_{0}-\check{D}_{0}\right)}{L \theta}, \quad \alpha_{1} \approx-\frac{S\left(\hat{D}_{1}-\check{D}_{1}\right)}{L \theta}, \\
\alpha_{2} \approx-\frac{S\left(\hat{D}_{2}-\check{D}_{2}\right)}{L \theta}-\frac{S^{3}\left(\hat{D}_{0}^{3}-\check{D}_{0}^{3}\right)}{6 L^{3} \theta},
\end{gathered}
$$

where the dispersions at the $\mathrm{F}$ - and $\mathrm{D}$-quadrupoles have been power expanded, respectively, as $\hat{D}=$ $\sum_{i=0}^{\infty} \hat{D}_{i} \delta^{i}$ and $\check{D}=\sum_{i=0}^{\infty} \check{D}_{i} \delta^{i}$. When $S \ll 12$, which is usually true because $S=2 \sin \frac{\mu}{2}$ and $\mu$ is the phase advance per cell, $\alpha_{1} / \alpha_{0} \rightarrow+\frac{3}{2}$ and reduces to $+\frac{1}{2}$ after chromaticities are corrected by sextupoles.

For an isochronous or quasi-isochronous ring, we must require the spread in $\eta$ for offmomentum particles to be small also. Therefore, $\alpha_{1}$ and $\alpha_{2}$ need to be controlled in addition to $\alpha_{0}$. In fact, first-order effect of sextupoles alters $\alpha_{1}$, that of octupoles alters $\alpha_{2}$, etc. For example, let $S_{n}=B^{(n)} \ell /(B \rho)$ be the strength of a thin quadrupole $(n=1)$, thin sextupole $(n=2)$, or thin octupole $(n=3)$ of length $\ell$ at a location where the horizontal and vertical dispersions are, respectively $D_{x}$ and $D_{y}$. Their first-order effects are [4] $\Delta \alpha_{0}=-S_{1}\left(D_{x}^{2}-D_{y}^{2}\right) / C_{0}, \Delta \alpha_{1}=-S_{2}\left(D_{x}^{3}-\right.$ $\left.3 D_{x} D_{y}^{2}\right) / C_{0}, \Delta \alpha_{2}=-S_{3}\left(D_{x}^{4}-6 D_{x}^{2} D_{y}^{2}+D_{y}^{4}\right) / C_{0}$.
The Hamiltonian describing the longitudinal rf phase difference $\Delta \phi_{\mathrm{rf}}$ is (Sec.2.3.1) [5]

$$
\begin{aligned}
H & =h\left(\frac{1}{2} \eta_{0} \delta^{2}+\frac{1}{3} \eta_{1} \delta^{3}+\frac{1}{4} \eta_{2} \delta^{4}+\cdots\right)+ \\
& +\frac{e V_{\mathrm{rf}}}{2 \pi \beta_{0}^{2} E_{0}}\left[\cos \left(\phi_{s}+\Delta \phi_{\mathrm{rf}}\right)+\Delta \phi \sin \phi_{s}\right],
\end{aligned}
$$

where $V_{\mathrm{rf}}$ is the rf voltage with synchronous phase $\phi_{s}$ and harmonic $h$, while $E_{0}$ is the on-momentum energy. If only the $\eta_{0}$ and $\eta_{1}$ terms are considered, the two series of distorted pendulum-like buckets in the top figure ( $\Delta \phi_{\text {rf }}$ vs $\delta$ with $\phi_{s}=0$ or $\pi$, see Ref. [2] for nonzero or non- $\pi \phi_{s}$ ) begin to merge to the middle figure when $\left|\eta_{0} / \eta_{1}\right|$ is lowered to

$\left|\frac{\eta_{0}}{\eta_{1}}\right|=\sqrt{\left|\frac{6 e V_{\mathrm{rf}}}{\pi \beta_{0}^{2} h \eta_{0} E_{0}}\left[\left(\frac{\pi}{2}-\phi_{s}\right) \sin \phi_{s}-\cos \phi_{s}\right]\right|}$.

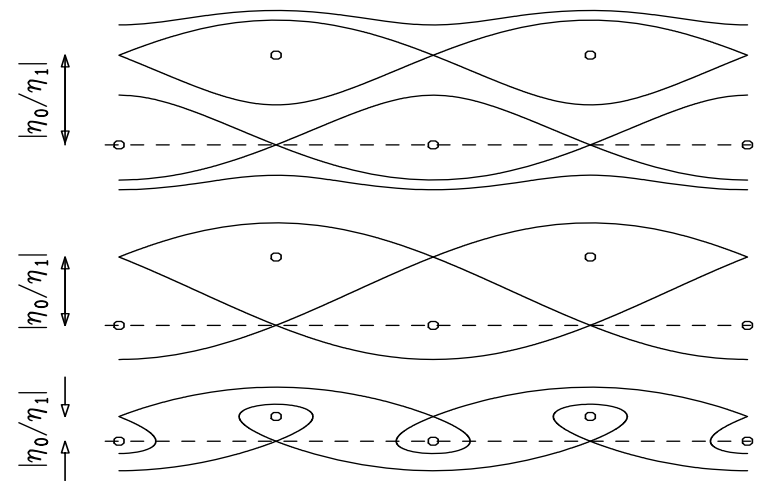

With further reduction of $\left|\eta_{0} / \eta_{1}\right|$, the buckets become $\alpha$-like (lower figure), which shrink to zero when $\left|\eta_{0} / \eta_{1}\right|=0$. The total bucket height $\left|3 \eta_{0} /\left(2 \eta_{1}\right)\right|$ is small. It is asymmetric in momentum spread and is susceptible to longitudinal head-tail instability. If the $\eta_{1}$ term is eliminated, the Hamiltonian will be dominated by $\eta_{0}$ and $\eta_{2}$ and the bucket becomes pendulum-like again [3]. If the Hamiltonian is dominated by the $\eta_{2}$ term alone, the kinetic term is similar to a quartic potential providing maximal amount of synchrotronfrequency spread and therefore Landau damping.

The first three orders of the slip factor can be extracted by measuring the synchrotron tune $\nu_{s}$ while changing the rf frequency $f_{\mathrm{rf}}$ : [2]

$$
\begin{gathered}
\nu_{s}^{2} \approx \frac{h e V_{\mathrm{rf}}\left|\eta_{0} \cos \phi_{s}\right|}{2 \pi \beta_{0}^{2} E_{0}}\left(1+\frac{s_{1}}{\eta_{0}}\left[\frac{\Delta f_{\mathrm{rf}}}{f_{\mathrm{rf}}}\right]+\frac{s_{2}}{\eta_{0}^{2}}\left[\frac{\Delta f_{\mathrm{rf}}}{f_{\mathrm{rf}}}\right]^{2}\right), \\
s_{1}=-\frac{2 \eta_{1}-\eta_{0}^{2}}{\eta_{0}}+\frac{1}{\gamma_{0}^{2}} \\
s_{2}=\frac{3 \eta_{2} \eta_{0}-2 \eta_{1}^{2}}{\eta_{0}^{2}}-\frac{\eta_{1}}{\eta_{0} \gamma_{0}^{2}}+\frac{3 \gamma_{0}^{2} \beta_{0}^{2}+2}{2 \gamma_{0}^{4}}
\end{gathered}
$$

Notice that $\Delta f_{\mathrm{rf}} / f_{\mathrm{rf}}$ is typically $\mathcal{O}\left(\eta_{0}\right)$. 
For the application of $\mathrm{THz}$ near-field imaging, $\mathrm{THz}$ spectroscopy, and others, bunch length compressed to the order of $\sigma_{\tau} \sim 1 \mathrm{ps}$ is desired. An obvious advantage is to store the bunch in the $\alpha$-like buckets, where the bucket half width, $\Delta \phi_{\mathrm{rf}} \approx\left|\eta_{0} / \eta_{1}\right| \sqrt{2 \pi \beta_{0}^{2} E_{0} h\left|\eta_{0}\right| /\left(3 e V_{\mathrm{rf}}\left|\cos \phi_{s}\right|\right)}$, is intrinsically narrow. Low-alpha operation modes have been implemented in many light sources. Essentially, $\alpha_{0}$ is reduced by making the dispersion outside the achromats of the ChasmanGreen lattice negative by scaling the quadrupole strengths. At BESSY II, a reduction from $\alpha_{0}=$ $7.3 \times 10^{-4} 100$-fold or even more is possible. [6] At SPEAR III, $\alpha_{0}$ has been reduced from $1.18 \times$ $10^{-3}$ 240-fold. However, smaller $\alpha_{0}$ implies shorter bucket height and therefore shorter beam lifetime. [7] To increase bucket height, sextupoles are used to minimize $\left|\alpha_{1}\right|$. For a more reliable operation of the machine, the low-alpha mode of BESSY II is compromised to $\alpha_{0}=3.5 \times 10^{-5}$ with zero-current rms bunch length reduced 5-fold to $\sigma_{\tau}=3.5 \mathrm{ps}$. For such an operation, no injection tuning of the optics is required and beam accumulates at a good rate up to a 200-bunch current of $5 \mathrm{~mA}$ with a 40-h lifetime. At SPEAR III, the $\alpha / 21$-operation mode incorporates a 21fold $\alpha_{0}$-reduction at $100 \mathrm{~mA}$ in 280 bunches with a 30-h lifetime, and a measured bunch length $\sigma_{\tau}=6.9$ ps. The shortest bunch length achieved has been $2.5 \mathrm{ps}$ at the single bunch current $3.5 \mu \mathrm{A}$, when $\alpha_{0}$ is reduced 240-fold. The beam lifetime is mostly limited by Touschek effect because of the short bucket height. When the bunch length is narrow enough, beam instability often occurs due to coherent synchrotron radiation.

\section{References}

[1] J. Wei, PhD thesis, SUNY, Stony Brook, (1990, rev. 1994)

[2] K.Y. Ng, Fermilab Reports FN-0578, FN-0852

[3] K.Y. Ng, NIM A404 (1998) 199

[4] D. Robin et al, PRE 48, 2149 (1993)

[5] K.R. Symon and A.M. Sessler, Proc. Int. Conf. High Energy Acc. (1956) p.44

[6] J. Feikes et al, EPAC 04, p.2291

[7] X. Huang et al, PAC 07, p.1308 